EPJ Web of Conferences 41, 12012 (2013)

DOI: $10.1051 /$ epjconf/20134112012

C) Owned by the authors, published by EDP Sciences, 2013

\title{
Heterodyne Detection of Electronic Optical Activity in Time- Domain: Single-Shot Chiroptical Spectrometry
}

\author{
Intae Eom ${ }^{1}$, Sung-Hyun $\mathrm{Ahn}^{1}$, Hanju Rhee ${ }^{1,2^{*}}$, and Minhaeng Cho ${ }^{1,3^{* *}}$ \\ ${ }^{1}$ Korea Basic Science Institute, Seoul 136-713, Korea \\ ${ }^{2}$ Graduated School of Analytical Science and Technology, Chungnam National University, Daejeon \\ 305-764, Korea \\ ${ }^{3}$ Department of Chemistry, Korea University, Seoul 136-701, Korea
}

\begin{abstract}
We demonstrate that an ultimately sensitive chiroptical measurement at single femtosecond pulse level can be achieved using heterodyne-detection techniques. These methods will be of use to observe molecular chirality changes in ultrafast time domain.
\end{abstract}

\section{Introduction}

Chiroptical spectroscopy, e.g. circular dichroism (CD), chiral luminescence, Raman optical activity, etc. has been widely used to study stereo-specific structures of chiral species. However, the conventional chiroptical methods utilizing both left- and right-handed radiations have fundamental limitations such as weak-signal, light-fluctuation and imperfect polarization switching problems, which have prohibited its further extension towards ultrafast time-resolved applications. To overcome such difficulties, we developed a new time domain approach [1-3], in which an impulsive chiroptical response signal, referred to as optical activity free-induction-decay (OA-FID) is characterized. A stark contrast to the previous differential measurement methods is that only a linearly polarized radiation (single polarization state, not both left- and right-handed ones) is used to excite the sample and the generated coherent OA-FID signals are detected using heterodyne method employing another local oscillator (LO) - note that this heterodyne detection scheme is very similar to that often used in coherent two-dimensional spectroscopic methods.

Although our initial infrared OA-FID measurements have been successfully performed [1-3], its extension to electronic applications at shorter UV-visible wavelength, which is an important frequency range for biological applications, should be challenging because that method relies on the interferometric detection. Recently, we developed two heterodyne detection techniques to overcome such phase fluctuation problem, i.e. self- and active-heterodyned OA-FID methods based on a simultaneous-and-pulsed detection scheme [4, 5]. The former method uses the fixed phase relationship between the signal and $\mathrm{LO}$, whereas the latter achieves power and phase fluctuation-free measurements on shot-by-shot basis. Here, we discuss those different heterodyne detection methods and demonstrate their experimental feasibilities. 


\section{Experimental}

The self- and active-heterodyned OA-FID techniques are depicted in Fig. 1(a) and (b), respectively, based on the simultaneous measurement of two parallel (achiral) and perpendicular (chiral) FID signals to the incident polarization. In both methods, the polarizing beam splitter (PBS) is used to spatially separate the FID signals combined with the internal (self-heterodyned) or external LO (active-heterodyned) and then simultaneously detect their spectra at CCD detector. Notice that the ratio of chiral to achiral FID field spectra directly yields circular dichroism (CD) and optical rotatory dispersion (ORD) spectra [1,2].

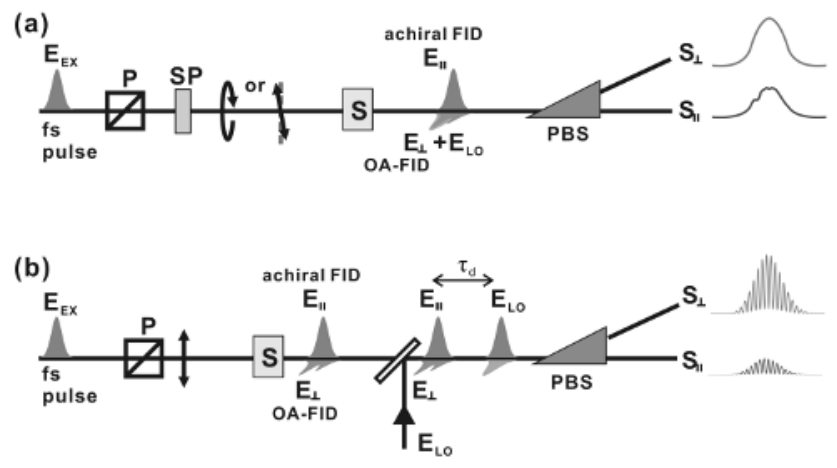

Fig. 1. Experimental layouts for (a) self-heterodyned and (b) active-heterodyned OA-FID measurement. P: polarizer, S: chiral sample, PBS: polarizing beam splitter.

For the self-heterodyned method, the incident radiation itself is used as the internal LO (minoraxis component) to interfere with the OA-FID. Because of the fixed phase relationship between the parallel FID and LO, the CD and ORD are separately measured depending on the incident polarization state (elliptical or rotated linear polarization). For the active-heterodyned method, on the other hand, the spectral interferograms between the FID and external LO are measured at a fixed time delay. In particular, the simultaneous measurement of the two interferograms in pulsed acquisition mode allows one to effectively eliminate the power fluctuation of the incident radiation and the phase jitter of the interferometer.

\section{Results and Discussions}

To demonstrate the active-heterodyned OA-FID technique, we considered the electronic $\mathrm{CD}$ and ORD of Ni-( \pm )-(tartrate) $)_{2}$ in water. Fig. 2(a) shows the heterodyned spectral interferograms of $(+)-$ form measured with only a single optical pulse. The standard Fourier transform spectral interferometry (FTSI) procedure is used to transform the real spectra of Fig. 2(a) to the complex ones. Then, the CD and ORD can be retrieved from the imaginary and real parts of their ratio (Fig. 2(c)). Interestingly, fairly decent CD and ORD spectra (dashed) are obtained even at the single-shot level. Examining three successive interferograms (inset of Fig. 2(a)) measured in pulsed acquisition mode, one can find that the phase jitter between the FID signal and LO is considerable as expected. However, the phase shifts shown in the chiral (upper of inset) and achiral (lower) FID are highly correlated with each other, indicating that the fluctuations equally contribute to the signals. Since such equal phase change cancels out by taking the ratio of the transformed spectra every pulse (selfreferencing), one can correct any phase and power noises caused by the fluctuations of the interferometer and/or radiation itself. 

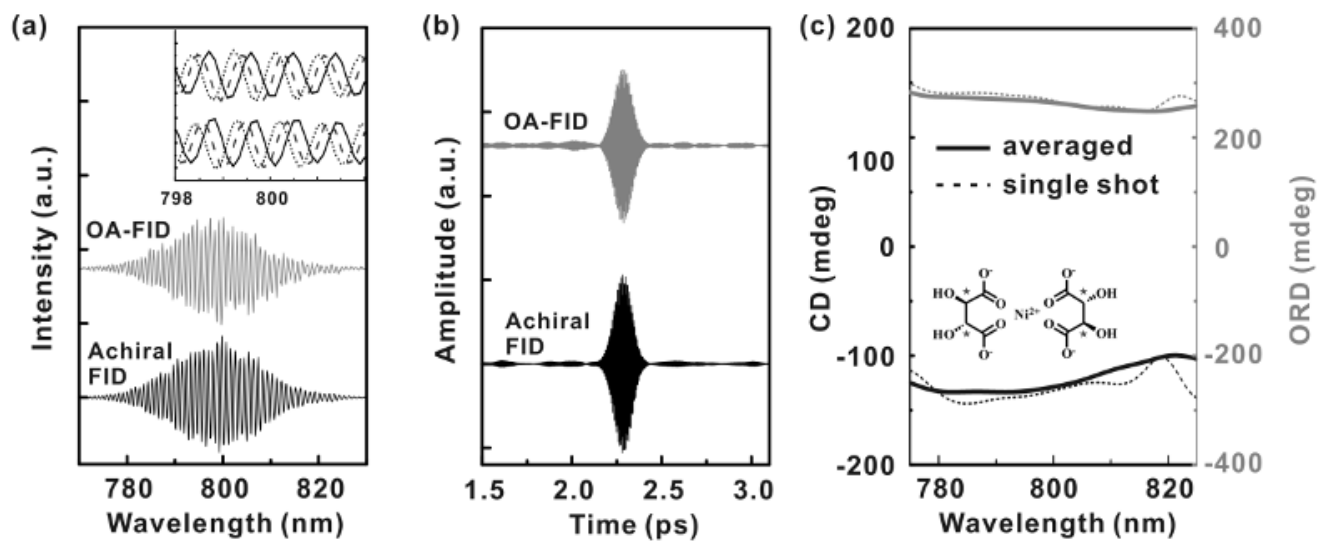

Fig. 2. (a) Single shot chiral (up) and achiral (down) spectral interferograms for Ni-(+)-(tartrate) $)_{2}$ in water. (inset: three successive chiral and achiral interferograms) (b) Chiral (up) and achiral (down) FIDs in time-domain. (c) Retrieved CD (black line) and ORD (gray line) spectra. Dashed line indicates the single-shot spectrum.

\section{Conclusions}

We discussed the self- and active-heterodyned OA-FID detection techniques based on the simultaneous detection scheme and demonstrated their single-shot detection capability. Thanks to the self-referencing advantage, our techniques enabled stable and reliable interferometric measurements even in UV-visible wavelength range. When combined with appropriate initiation methods such as temperature jump, $\mathrm{pH}$ jump, photo-isomerization, and so on, these novel approaches will be powerful tools for ultrafast structural dynamics studies of chiral biomolecules.

\section{References}

1. H. Rhee, Y.-G. June, J.-S. Lee, K.-K. Lee, J.-H. Ha, Z. H. Kim, S.-J. Jeon, and M. Cho, Nature 458, 310 (2009)

2. H. Rhee, Y.-G. June, Z. H. Kim, S.-J. Jeon, and M. Cho, J. Opt. Soc. Am. B 26, 1008 (2009)

3. H. Rhee, J.-H. Choi, and M. Cho, Acc. Chem. Res. 43, 1527 (2010)

4. I. Eom, S.-H. Ahn, H. Rhee, and M. Cho, Opt. Express 19, 10017 (2011)

5. I. Eom, S.-H. Ahn, H. Rhee, and M. Cho, Phys. Rev. Lett. 108, 103901 (2012) 